\title{
The Bugis Chronicle of Bone: Bugis text
}

$[p \cdot 1]^{1}$

Ianaé sure’ poada-adaéngngi| tanaé riBoné| enrengngé mangkau’ riBoné| angkanna rirapié méngkalinga| napauwé tomatowaé|

Tania upomabusung| tania upomawedda-wedda| tekkumatula poada| aseng tolébba| nasekko' rumasa sélo-sélo| ana' tolebbi'| Aga uwasimammémeng| kuinappa lakke'-lakke’| wija senrima mangkau'|

Ia garé puttana arung ménréé| riGaligo| déna riaseng arung| Aga tennassiseng tauwé| siéwa ada| Sianré-balémani tauwé| Siabelli-belliang| Déna ade'|apa'gisia riasengngé bicara| Riasengngi pitu-tturenni| ittana| dé arung| Sikuwa toniro| ittana| tauwé tessise-ssiéwa ada| tekké ade'| tekké bicaral

Naiamani ammulanna| nangka arung| Engka séuwa esso| nasianré billa’é| letté| <m>péwattoni tanaé| Riasengngi| engkai sipasa makkua| Naia pajana billa’é| letté| $<\mathrm{m}>$ péwang tanaé| takko' engka tau rita| woroané| ritengngana padangngé| masangi $<\mathrm{m}>$ puté| Jajini sipulung tauwé| tasséwanua| tasséwanua| Iana riassiturusi| ritau maégaé| masengngéngngi| tomanurung|

Jajini passéuwa tangnga'| tau maégaé| Naia nassiturusi $\mid<$ m>pokke’éngngi aléna| llao ritauwéro| nasengngé tomanurung| Lattu’i koria| Makkedani tau tebbe'é| "Iana mai kilaowang riko| Lamarupe’| amaséannakkeng| aja’na

1 Page numbers refer to the original manuscript, NBG 101. 
muallajang| Mutudanna ritanamu| Naikona poatakkeng| Élo'mu élo' rikkeng| Napassuromuna kipogau'| Namau anammeng| na patarommeng| mutéaiwi| kitéaitoisi| Rékkua monromuno mai| naiko kipopuang|"

Puraikua| makkedaniro| riasengngé| tomanurung| "Madécénnami| Naianasia| upoadadako| temmakulléa' arung| apa' atawa'sia| Naérékkua maéloko| makképuang| engkaro puakku’| Iaro mupopuang| rékkua maélo’kkeng|"

Makkedani tomaégaé| "Mappékkoni kisseng| kipopuang| tekkitaé|”

Makkedani riasengngé| tomanurung| "Rékkuwa maélo’ tonge-ttongekko| upaitaio|"

Makkedani tomaégaé| "Maélo' wégang nakkeng naé tabbuluko mamasé| lalengeng nakkeng|”

Puraikua| rilalengnganni tau maégaé| llao riasengngé riMatajang| Sianréni paimeng letté| wéro’é|

[p. 2]

Aga lettu’i riMatajang| napolé itani| tomanurungngé| tudang ribatu lappaé| Sangi $<\mathrm{n}>$ ridi eppa situdangeng| Watanna arungngé| séuwa pajungiwi| pajung maridi| séuwa $\mathrm{pa}<\mathrm{m}>$ piriwi| séuwa tiwirangngi| salénranna|

Apa' llaoni kuria| ritomanurungngé| Makkedani Tomanurungngé| "Engkao Matoa|"

Makkedai Matowaé| "Io Puang|"

Inappani naisseng tomaégaé| makkedaé matoamua palé| tasengngé arung| Makkedani to riasengngé manurung| "Iasiaro puakku'|"

Purai kua llaoni| tau maégaé| ritomanurung sangi $<\mathrm{n}>$ ridié| Nakkedana tau maégaé| "Iana mai kilaowang ridi' Puang| maélokkeng tamaséang|tamaradde'na mai ritanata'| Aja'na tallajang| Idi'na kipopuang| Élo’mu élo' rikkeng|passuromua| kua| Namauna <a>nammeng| enrengngé pattarommeng| mutéaiwi| kitéaitoisi| rékkuwa tudammuni' mai| Naikona poatakkeng| Mudongiri temmatipakkeng|"

Makkedai Tomanurungngé| "Teddua nawa-nawao| temma’balléccoko|" 
Purai kua sikadonni| adanna tomanurungngé| tau maégaé| Rilékke’ni| Manurungngé| llao polé| riBoné| Ianaé Manurungngé| mangkau’ riBoné| Ripatettongang langkana| Tépui langkanaé ripaténi Manurungngé| ttudang rilangkanaél

Naia Manurungngéwé| tettadapi' méngkalinga| aseng rialéna| Gau'namenna ritellakangngi| Rékkua naitai lompo'é| napenno tau| natiroi naissemmenni ballalo| makkedaé| sikuwa tauro| makkedaé sikuwaro tau² Aga naiamenna ritellarangngi| tania kupomabusung| Matasélompo' é3

Matasilompo'éna | ma'bawiné riToro'| siala Manurungngé riToro'| tania kupomabusung| Nana' La Ummasa'| tania kupomabusung| enrengngé riasengngé| tania kupomatula| La Pattanrawanua| asenna| Limai sijajing| Naia saisáé| koi mmonro riattoriolong rilullungngé| Ia muasi naripau risure'éwé| allapi-lapirenna| gau'é| riBoné| tassilapi-tassilapi|

Naia gau'na Manurungngé| Mangkau'é riBoné| iana mula patettong| riasengngé| Mappololéténg| Iana ppadengngiwi| assisulu'-sulurenna| akkéanungngé wali-wali tomakkéanué| Pada maradde' manenni ritauwé| Takkalaé mattiwi| tenriolanna siabbicarang| Iatona patettong Rapang Bicara| enrengngia Ade'| Naiana| riolai| Iatona Manurungngéwé| punna Baté Woro $<\mathrm{m}>$ porongngé|

Naia genne'-

[p. 3]

-na| patappariama| mangkau' riBoné| napasipulunni toBoné| Mapparénnaiwi| nakkeda "Tudanno m<a >i| Aja" mumarullé| Ianaritu ana’ku| riasengngé La Ummasa'| Iana ttolawa'| Iatona upattenning akkuluadangetta'|"

Puraikua billa’ni| letténi| Takko’ déni rita| ritudangenna| Manurungngé| ia dua mallaibini| laona ${ }^{4}$ ritai| Paju $<$ n>ridié| Détoni rita rionronna| Salénrangngé détoni| Ripatetto-mmutoni ballalo| mangkau'| tania kupomabusung| riasenna Puakku'| La Ummasa'| Nadétona pajung riBoné|

2 The close repetition of this phrase is a scribal error. In the manuscript, the second version of the phrase has been crossed through. Matthes also notes the readings of NBG 100 and NBG 99 to explain the passage.

3 Sélompoee. The usual spelling is Silompo’é, as follows immediately.

4 The first aksara of laona is illegible, but this reading is supported by other manuscripts. 
2

ManurungngériMatajang poana'i|LaUmmasa'|riasettoiToMulaiépanreng| Naia mallajannana Puatta'| ia dua mallaibini| tania kupomabusung| La Umasa'na| mangkau' riBoné| Pawélaimani| nariaseng To Mulaiépanreng| Iana mangkau'| kalio'namani| ricinaongangngi| Rékkua engka nalaowi mappeddi'-essomani| Dénnana pajung riBoné| Iatona riaseng| Panrébessié| Ripujito mainge'| Riasettoi maléleng| Riasettoi matanang|

Nallakkaina| anádaranna Aru $<\mathrm{m}>$ Poné| riasengngé| Pattanrawanua| siala makkarungngé riPalakka| riasengngé| La Pattikeng|

Iatonaé mangkau' riBoné| nabétai Biru| nabétai Cellu| nabétai Malloi' nabétai| Anrobiring| nabétai Majang| Iatona sisala| ipa'na| makkarungngé riPalakka| riasengngé La Pattikeng| Nasiwangungang musu' maripa’| Natellumpuleng mammusu'| tessicau'| nasiajje'| mennang| Iatonaé| dépakua riaseng| rajanna riBoné| tanréna uwatanna|

Nadé ane'na pattola| To Suallé mua napoana'| enrengngé| To Salawaka| naé pa'banua mua inanna| Aga naissengngi| mattampu' ana'daranna| mallakkaié riPalakka| Nalao ti<n $>$ roini| riaseng| ripaitaianni| inappani massau ininnawana| Apa' naissenni| manguriwe’| ana'daranna| mallakkaié riPalakka| nata $<$ m>paini toSuallé| enrengngé toSalawaka| Nakkeda "Lao sao urai' masiga'| riPalakka| apa' riasengngi manguriwe'na| anri'ku'| Naé' rékkua| alepperrenni| anri'ku’| pariappoppa-mmuani| raraé| mutampui| muwawai llao mai alau| masiga'| Kuapi mai risappe' lolona| kuatopi mai| ribissai|"

Madaka-rakatonisia| To Suallé| To Salawaka| llokka masiga'| Lattu’i riPalakka| matou'-tou'ni té' masiga'| risalassa'é| Tettuda-

[p. 4]

-ttopa| To Suwallé| To Salawaka| Nalepperenna makkunrainna| Arungngé riPalakka| Woroané ana'na| Manganro-manai'-maneng gemme'na| Mattou'-tou'menni To Suallé napariapoppangngi| raraé| nata $<\mathrm{m}>$ pui| risa $<\mathrm{m}>\mathrm{pu}$ reppung| Nalaowangngi| alau riBoné| Naé déi arungngé riPalakka| nariala ana’na| 
Apa' lettu’i riBoné| ripattou'-tou'ni té' rilangkanaé| Inappani risappe' lolona| inappato ribissai Anádarannana Aru<m>Poné| riasengngé I Samateppa| risuro matuwoi| I Samateppana maruppengi| anauréna| Riobbira-mmémenni ritoBoné| wenni séwenni| makkedaé "Sipulukko baja| ttiwi' paréwa musumu|"

Papai bajaé| engka manenni toBoné| sakke’ paréwa musu’| Ripadau’ni| Woro $<\mathrm{m}>$ porongngé| No'ni Aru $<\mathrm{m}>$ Poné| ribarugaé| Makkedai| Aru $<$ m $>$ Poné| “Ia mennang kupasipulungakko| to Boné|LaSaliwuro asenna ana'ku'| Kerra $<\mathrm{m}>$ pélua' pattellarenna| Upaléssoriniro akkarungengngé riBoné| Iatonaro ana'ku’| kupatenning uluadaé| napawarekkengiéngnga' Puwatta'| nainappa mallajang|"

Sama kadoni to Boné| ia maneng| nainappana mangngaru| Nassuro mutona ténai bissué| Naripatettong ballalo| pabbinru’é esso sésso| Narilanti'na Puatta'| Kerra $<\mathrm{m}>$ pélua’| riamauréna| pitungngesso| pitu $<\mathrm{m}>$ penni| Naiamanisi| garé' taué| riwaéngngi raraé iana riaddojang| pitungngesso| pitu<m>penni| Apa’ genne'ni pakkawarué| inappani riwélésu lolona| Purai riwélésu érunna| no’ni risalassaé| Puatta’ Matowaé|

Puatta' Kerra $<\mathrm{m}>$ pélua'| riaseng Aru $<\mathrm{m}>$ Poné| Iana mmonro rilangkanaé| Inaurénana| riasengngé| I Samateppa| tomatowaiwi| Naia Puatta' Matowaé| rékkua engkana maélo'| llaowi| massuroni manai' riana’na| makkedaé| “Tésao manai| ripuammu| Muakkeda engka maélo' nalaowi Puatta'| Assurosao <m>pawai|”

Iatonasia garé" riwaéngngi raraé| makkeda "Laosaoromi muta $<\mathrm{m}>$ paiwi| tau nalaosa ttiwi'i Puatta'|" Makkuaniro garé gau' na Puatta’| rékkua engka nalaowi| namunagi-namunagi| gau'na Puatta’ Matowaé|

Naseppulo pitu taunna| napaléssori akkarungeng| anauréna| nateppani lasa maserro| Puatta' Matowaé naiana mpawai| Nariasessi Puatta'| Mulaiépanreng| 
Puatta’ Mulaiépanreng| poanauréi Puatta' Kerra<m>péluwa'| Puatta’na Kerrampéluwa'| Mangkau’ riBoné| Makkaru-mmémenni rituo-

[p. 5]

-na amauréna| apa' séwennimua| jajinna| naripaléssori akkarungeng riBoné| rijajinna| Rilanti' matoni| na To Sualléna mmawingi| na To Salawakana Makkedantana| Naé' rékkuwa| engka bicara namaterre'| napettui Tomabbicaraé| ripatén ni manai' rilangkanaé| nakuwana sipakkeda| $\mathrm{Na}$ To Suwallénna $<$ n>riwai raraé| nasipakkedana tauwé| wali-wali| $\mathrm{Na}$ To Salawakana sauriwi adaé| wali -wali|Naiyatona riwaéngngi raraé|iyana pannessai|asalanna tauwé|tau salaé| Riasettonisia| Pettu Bicaranna| Raraé|

Apa' marajani| Kerra $<\mathrm{m}>$ pélua'| tappini makkunrai| Inappani llao riPalakka| sita ncajiangngéngngi| Inappaniro sita| apa' lettu’i riPalakka| ritoanani rincajiangngéngngi| Narimanarinna|Naiatonaro| narimanarenna pasaé riPalakka| Nariwawana pasaé riPalakka llao riBoné| Nakuwa tauwé| riBoné mappasa|

Iatonaro llaona riPalakka rincajiangngéngngi|naripabbawinéna| siala sapposisenna| riasengngé| Wé Tenriroppo| ana' pattolana Arungngé riPaccing| Ia poana’i| tania kupomabusung| riasengngé| Wé $\mathrm{Be}<\mathrm{n}>$ rigau'| Daéng Maroa pattellarenna| Iamuto riaseng Makkale $<\mathrm{m}>$ pié| Riasettoi Bissu riLale $<\mathrm{m}>$ pili'| Iana ripakkarung riMajang| Nariséséng| toBukakaé| saisa'| nariwawa riMajang| mmonro| Naiatonasia| napotauto Makkale $<\mathrm{m}>$ pié| Naripatettongina Saolampé| riBoné|nariaseng Lawélareng| Napoasettoni Makkale $<$ m>pié Puwatta’ riLawélareng|

Naia Puatta' Kerra $<$ m $>$ péluwa'| ripuji malessi|Ripujitoi maléleng| Ripujitoi mapato lao $<\mathrm{n}>$ ruma| Ripujitoi malabo $\mid$ Tenriase $<$ m $>$ pégasa tomacca| Dé’ cau'i awaraningenna| Mau garé inappa jajinna dé mémessa napoléiwi takkini'| lettu' rimatoana| Tennaisseng mémessa riaseng| péneddingenna riasengngé takkini'| Aga naia poasengngi| Passoddo’ Wakkaé|

Iatona arung mmula massuro poada| ada-passokkang ribalié| rékkua maélo’i mosengiwi balié| Apa' ia riasengngé tuppu-batu| enrengngiya timu-timu napogau' mémessa| arung rioloé| ménréé riGaligo enrengngé massuroé| 
Ianaé Kerra $<$ m>pélua'| makkarung nawinru’ baté Cella’ dua| Naia Cella’é| dua Woromporongngé| séuwa ri<a > béona| séuwa riataunna|Natawa telluni toBoné aléna| Sétawang maccinaongiwi Cella’é| sitawang maccinaongiwi Woromporongngé| Naia Woromporongngé maccinaongiéngngi| toMajangngéna| enrengngé toMata-Angingngé| enrengngé toBukakaTengngaé| enrengngé toKawerrangngé| enrengngé toPalléngorengngé| enrengngé to

\section{[p. 6]}

-Mallarié| Namatowaéna riMatajang $<\mathrm{m}>$ pawai| Naia maccinaongiéngngi Cella'é| Riataunna| Woromporongngé| toPaccingngé| enrengngé to Tanété| enrengngé toLémo-Lémoé| enrengngé toMasallé| enrengngé toMacégéé| enrengngé toBélawaé| naKajao Ciunna mpawai| Naia maccinaongiéngngi Cella'é| $\mathrm{Ri}<\mathrm{a}>$ béona Woromporongngé| toArasengngé| enrengngé toUjungngé| enrengngé toPoncéngngé| enrengngé toTảé| enrengngé toKatumpi'é| enrengngé toPadaccengngaé| enrengngé toMadello'é| naKajao Arasenna mpawai| Naia watanna ArumPonél lélé uléni Aru $<\mathrm{m}>$ Poné| mappattuju|

Puatta’ Kerra $<\mathrm{m}>$ pélua'| bbétai Palléngoreng| Sinri| Anrobiring| Iatona bbétai Lé $<$ m>pang| Mellé| Iatona bbétai Sancénreng| Cirowali| Apala| Bakke'| Tanété| Attassalo| Soga| Lampoko| Lémoape'| Bulu'-Riattassalo| Parippung| Lompu|

Iatona mangkau'| napattau-séuwai| toBoné| na toPalakkaé| Ana’ni tanaé riPalakka| riBoné| Nangkana mmutama riBoné| Lima $<m>$ panuaé|Rilauale'| maddaoangngi tanana riBoné| Nangkatona Arungngé riBabauwaé| riasengngé La Tenriwasu| sita ménéttu-eppona| napaddaowi tanana| Napattau-séuwani ArumPoné| toBoné toBabauwaé| Ana’ni tanaé riBabauwaé riBonél Nangkasi mutama arungngé riBarebbo| paddaowi tanana riBoné| Ana’ni tanaé riBarebbo| riBoné| Nangkatona muttama' Arungngé riPattiro riasengngé La Paworong| sita Aru $<\mathrm{m}>$ Poné| apa' sipoipai| paddaowi tanana| Naripatuda-ppalilina| tanaé riPattiro| riBoné| Nangkatona muttama Cinennung| Ureng| Pasé<m>pe'| maddaowangngi tanana| Naripatudanna Tellu $<$ m $>$ panuwaé|

Éngkatoni arungngé riKaju riasengngé La Tenribali| paddaowi tanana riBoné| naripatudappalili'na Kaju| Nawata'-mutona duta| Arungngé riKaju| riana’na ArumPoné| ritellaé Makkalempié| tania kupomabusung| Wé $\mathrm{Be}<\mathrm{n}>$ rigau' aseng $<$ n $>$ rialéna| Naritangke'na Arungngé riKaju| riArumPoné| Nainappana ppenning riwanuwanna| Arungngé riKaju| 
Nakuwananaro mai riwanuwanna| nainappana lao botting| riBoné| Nallaibiningenna ArukKaju| riasengngé La Tenribali| ana’ na Aru $<\mathrm{m}>$ Poné| ritella'é Makkalempié|

Nangkatona ArupPonré| maddaoangngi tanana riBoné| Nangka manettona mmuttama| Aséraé Baté| riAttangngale’| enrengngé Aséraé Baté| riAwangngale'| maddaoangngi tanana riBoné| Anảni riBoné Aséraé Baté| riAttangngale’| Aséraé Baté riAwangngale’|

Kerrampéluảna Mangkau’ riBoné| nabéta maggulilingngi wanuwaé riBoné|

[p. 7]

Iatona arung riaseng| maserro pakarajai tomatoanna| Iatona Arung Mangkau'| napassu'i ata rialéna| nataroi riPanyula' | nariasenna toPanyula’é| Naia ata nalollongngé| angka makkarunna| kui nataro riLipenno| $\mathrm{Na}$ toPanyula'éna| silaong toLimpennoé| makkasiwiangang balé| Iatona mpisé rékkuwa llao mallopiwi ArumPoné| Iatona pampulé| rékkuwa llao mabélai ArumPoné|

Naia genne'na pituppulo duwa taunna| mangkau'| napasipulunni toBoné| séllili| Nakkedana ArumPoné| "Ia mennang kupasipulungakko| matowana’| useddittoni aléu' madodong| Naé maéloka’ mmitao maréwangeng|"

Puraikuwa| samakadoni toBoné| Massa<ppa >matoni tauwé| esso|Narapini| esso rita $<$ n $>$ raé| maréwangenni tauwé| Ripadau'ni Woro $<$ m $>$ porongngé| Purai maréwangeng tauwé| natowanani toBoné| séllili'| Purai manré tauwé| makkedani ArumPoné| "Iatopa mennang uwakkatta poadakko| toBoné| Iatu ana’ku’ riasengngé Wé $\mathrm{Be}<\mathrm{n}>$ rigau'| uwélorang makkarung riBoné| rékkuwa matéa'| Iatonaritu kupawarekkengi uluada| napatiangngéngnga' Puatta’| Mulaiépanreng|

Purai kuwa ssoro'ni tauwé| Naséwennimua purana mappaseng| nateppani lasa| nasia’mutona 
Puatta' Kerrampéluwa'| napoana’ni Mallajangngé riCanai ${ }^{5} \mid$ Ia pawélainna Puatta’ Kerrampéluwa'| Makkalempiéna Mangkau’ riBoné| Ia riappasengngang rincajiangngéngngi| tania kupomabusung| Wé $\mathrm{Be}<\mathrm{n}>$ rigau' aseng rialéna| Daéng Maroa pattellarenna| Riasettoi Arungngé riMajang| mangkau’mani nariaseng ArumPoné| Ripuji kénawa-nawa|

Inappani duwa-ttaung| nawette' rara| Naripatangngarinna rincajiangngéngngi| Iana siala ArukKaju| riasengngé La Tenribali| Asera ana’nana| naé duwamui ripauttamá risure'éwé| Naia ana’na pitué| kuisia mmonro riattoriolong rijori'é| Naia engkaé risure’éwé| tania kupomabusung| riasenna Puakku’| La Tenrisukki' enrengngé La Tenrigora| ana' naé

Makkale $<$ m>pié| mangkau'| nasuroi Arungngé riKatu $<$ m $>$ pi| riAttassalo| riasengngé La Dati'| mméllau' mmelliwi bulu'é riCina| Aséra pulona tédong tenrilase'| Naripabbellinna| Nangellina| Puatta' Makkale $<\mathrm{m}>$ pié| Bulu'é riajanna Laliddong| Tellu-ppulo tédong naelliangngi| Puraikuwa| nassurona mmonroiwi Bulu'é riCina| Nassurotona palla'i| Nassurotona llaoiwi Bulu’é riajanna Laliddong| naellié|

Naduwa-ttaunna| napalla' Bulu'é riCina| enrengngé nauma| galungngé riajanna Laliddong| nariakkecca'

[p. 8]

-na pallao $<$ n $>$ rumana| enrengngé palla’nal mmonroé riCinal ritoKatum $<\mathrm{m}>$ pié| Massuroni ArumPoné painge’i| Arungngé riKatu $<\mathrm{m}>$ pi

Natell $\mathrm{u}<\mathrm{m}>$ pulemmua poléna surona| ArumPoné painge'i $\mathrm{La} \mathrm{Tu}<\mathrm{m}>\mathrm{pi}^{\mathrm{7}} \mid$ nariwunona Jennanna ArumPoné| Natérini Katu $<\mathrm{m}>$ pi| Naribétana Katumpi' ritoBoné| Rirappa esso sésso| Narialana galungngé rilauna Laliddong| Enrengngé riawanna Laliddong|

5 The manuscript reads Canai, but as Matthes has written above it, the correct reading is certainly Cina.

6 ana'naé. There is a pallawa before this word, but none after it. Matthes has written in, and clearly prefers, the reading of NBG 99, ianaé, which then forms the first word of the next paragraph.

$7 \quad$ La Tumpi here means the ruler of Katumpi'. 
Naia panyu $<\mathrm{m}>$ parenna ArumPoné riasengngé La Tenrigora| iana rimanariang riMajang| enrengngé riCina| Iana riaseng La Tenrigora| ArucCina| riasettoi ArumMajang|

Naia ana’na Aru<m>Poné| tania upomabusung| enrengngé upomatula| riasengngé La Tenrisukki'| Iana ripaléssori akkarungengngé riBoné| Narilanti'na rincajiangngéngngi| Nariasenna Aru $<m>$ Poné| tania upomabusung| riasenna La Tenrisukki'| Seppuloi-sétaung naripaléssori akkarungeng rincajiangngéngngi|

Naia purana Puatta' Makkale $<$ m $>$ pi'é| nalanti'ni ana'na| napatudanni rilangkanaé| Nalaonasa riCina| mmonro silaong ana' panyomparenna| riasengngé La Tenrigora|

Napata-ttaung mmonro riCinal Makkale $<\mathrm{m}>$ pi'é Nangka séuwa esso| natakkau' ménrémua Makkale $<$ m $>$ pi'é| riarakéanna| Nakuwana riarakkéangngé| tudangngi jarasana| Naé' engka naseng torioloél Api Déwata| Natakko' <e>ngkamuaro mai| ma’balute' ribolaé| addénémmua garé naola| Lattu'i garé manai' ribolaé| ma'balute' ulé|Tési manai riarakkéanna| Peddénisia $<\mathrm{A}>$ pi Déwataé| détonisia rita Makkalempi'é| Riasenni Mallajangngé riCina|

5

Mallajangngé riCina| poana’i ritellaéMappajungngé| tania kupomabusung| La Tenrisukki' Mangkau' riBoné| Pata-ttau-mmémenni purana ripaléssori angkaukeng| rincajiangngéngngi| napawélai Mallajangngé| Nasialana sapposisenna| riasengngé Wé Tenriso $<$ ng $>$ ké| Naiasi ncajiangngi tania upomabusung| riasengngé La Wulio| ritella’é Boto’è $\mid$

Naengka manenna Arung Sébulu'é| mmuttama’ riBoné| maddaoangngi tanana| Ripatuda-ppalilina|

Iatona Mangkau' riBoné| naengka Datué riLuwu| ritellaé Déwaraja| ttériwi Boné| Nakuwa| riattanna Cellu| ssoré Luwué| Nakuana ttaro tudang| Naia purana| sisokkang rilalengngé lluanni| makku< $<$ n raié saisa' na toriAttassaloé $\mathrm{tti}<\mathrm{n}>$ rosiwi| massu maniang riAttassalo| ridenniarié|

8 The name is given here, and on p. 9, 11. 21 and 22, as Boto'é. This is clearly a mistake for Botéé, meaning 'the Fat Man', which occurs frequently below and is specifically justified in the text. 
mmose-ttonisia-romai| luwué| Maélo’ni naoloi| osonna| Kkotonisia toBoné| riBiru| paranrung ttudang| Namappappana bajaé| makkarimatani luwué| naitani

\section{[p. 9]}

makku<n>raié| rilauna $\mathrm{A}<\mathrm{n}>$ robiring| rilalengngé| Ianakkajuru'-juru'ki| Mattebbanni toriAttassaloé| luwué| Riarupai| luwué ritoBoné| Ripalari salani| luwué| Rialani pajunna Datué riLuwu| Iamua tennariwetta| Datué riLuwu| Engkanamua Aru<m>Poné| paleppengngi taué| nakkeda| "Aja' muwettai| watanna Datué riLuwu|"

Nariti<n $>$ rona llao alau| lattu' rilopinna| Engkamani madduappulo lattu' rilopinna| Datué riLuwu| Nalopi baiccu'menna| natuju naola| Iana natonangngi llao riwanuwanna| Ianaro nangkasi pajung paimeng riBoné| Naé pajung cella'sia| pajunna Datué riLuwu| rialaé| Agana ritellana| tania kupomabusung| La Te $<$ n $>$ risukki' Mappajungngé|

Iatona mangkau' riBoné| nasisala toMa $<\mathrm{m}>$ pué| toBoné| Nasiwangungang musu| Nasiosengngenna| Nakua siduppa riattanna Itterrung| Naribuanna toMa $<\mathrm{m}>$ pué| naripalattu' riwanuwanna| Nassu'na ArumMa $<\mathrm{m}>\mathrm{pu}$ manyo $<\mathrm{m}>$ pa| sorongeng sebbu-kkati| "Élo’mu élo'| Aru $<\mathrm{m}>$ Poné| kko temmupassara-mmenna ana'ku' pattaroku'|”

Makkedani Aru<m>Poné| "Kupalimuo| ArumMampu| mutudappalili riBoné| Temmacaddiko riBoné| Temmuacinnaiang ulawettasa'| pattola mala $<$ m $>$ pé $\mid$ wara $<$ m $>$ para-mmala $<$ m $>$ pé $\mid$ Mupasengngangngi torimu $<$ n $>$ rimmu|"

Nainappana ritelli' ArumMa $<\mathrm{m}>\mathrm{pu} \mid$ Purai ritelli' ArumMa $<\mathrm{m}>\mathrm{pu} \mid$ $\mathrm{e}<\mathrm{n}>$ rengngé lilina| lisuni Aru $<\mathrm{m}>$ Poné riwanuwanna|

Naduappulo pitu-taunna mangkau'| nateppai lasa| Napaddeppungenna toBoné| nakkeda "Maserro lasaku' |naé dékkua matéa'| ianatu ana'ku| riasengngé riLa Ulio| ianatu tolawa'|"

Purai mappaseng| massialamotoni| 
6

Mappajungngéna poana'| Matinroé rItterrung| Naia pawélainnana| Mappajungngé Botéési Mangkau'| riBoné| Apa' ia riappasengngeng| ri<n>cajiangngéngngi| tania kupomabusung $\mid$ riLa Ulio' ${ }^{9}$ aseng rialéna| Riasengngi malolomupa| namaloppo| Tallebbi pituisia $\mathrm{pa}<\mathrm{m}>$ puléna| Nassisulléngi pa $<\mathrm{m}>$ puléna| Aga naritellana Boto’é|

Ianaé arung mapparessang| mappattuju| Iatona riaseng mélori ssawung| riaseng matanang|

Ianaé Boto’é| riaseng siala ana’na| Arungngé riPattiro|ritellaé Magadingngé| riaseng Wé $\mathrm{Te}<$ n $>$ riwéwang| $\mathrm{Da}<\mathrm{n}>$ raé pattellarenna|

Ana’na Magadingngé| mapowawinéi Botéé| najajina| tania upomabusung| riasengngé $\mathrm{La} \mathrm{Te}<\mathrm{n}>$ rirawé| ritellaé Bongkangngé| Najajitona| tania upomabusung| riasengngé La Icca'| Najajitona| tania upomabusung| retella'é $\mathrm{Te}<\mathrm{n}>\mathrm{ripauwang}$ najajitona| tania upomabusung| riasengngé| I Lémpe'|

Ianaé| Botééwé| arung mmula riranreng| riKajao Laliddong| Iato[p. 10]

-na| arung makkuluada| Karaéngngé riGowa| riasengngé Daéng Mata $<$ n $>$ ré| Iatona nariaseng adaé| sitettongenna| Sudeng| La Téariduni| Iatona bbétai| Datué riLuwu| $\mathrm{mo}<\mathrm{n}>$ rona $\mathrm{riCe}<\mathrm{n}>$ rana| nasilaong karaéngngé| riasengngé Daéng Bonto| ana’na Daéng Mata $<$ n $>$ ré| Nalanni Lise’ Karaéngngé| malani Lappa Aru $<\mathrm{m}>$ Poné|

Ma'bawinétoni riMa $<\mathrm{m}>$ pu Botéé| siala Wé Tenrigau'| ana’na ArumMa $<\mathrm{m}>\mathrm{pu} \mid$ riasengngé Daéng Palimpu|

Naiatonaé Botéé| Mangkau’ riBoné| nalao mai| Karaéngngé riGoa| namula malla’éngngi| tanaé riBoné| Iana nariaseng| nasitudangeng| Karaéngngé riGoa| Aru <m>Poné| riattanna Laccokkong| Nasiwuno toGowaé| toBoné| Naé rékkowa toBoné pawetta| Karaéngngé riGoa| passa $<\mathrm{m}>$ puriwi| Naé rékkowa toGowaé| pawetta Aru $<\mathrm{m}>$ Poné passa $<\mathrm{m}>$ puriwi| Iatona Mangkau'| narisilaongang| riKaraéngngé riGoa| Nalai sebbu-katinna toWajo'é| kuwa riasengngé riTopacceddo|

9 It is not clear why the copyist writes ri-before the name La Ulio here and in the previous chapter. 
Naia genne’na| duappulo lima-ttaunna| Mangkau' riBoné| ripasipulunni toBoné| nakkeda "Maélo’ka' mennang| paléssoriwi akkarungengngé| ana'ku'| riasengngé $\mathrm{La} \mathrm{Te}<\mathrm{n}>$ rirawé|"

Samakadoni toBoné| nalanti'ni ana’na| pitungngesso pitu $<$ m $>$ penni| Purai nalanti' ana’na| nonno'ni risalassa'é| Puatta Botéé| Maddua wanuwani| Engkana llao riMa $<\mathrm{m}>$ pu ribainéna| engkana llao riBoné|

Iana Botéé| magelliwi ana’uréna| riasengngé La Pau<n>ru| Nagellitoi sapposisenna| makkarungngé riPaccing| riasengngé La Mulia| Maélo’ mappawakkangangngi aléna| ritoMa $<$ m>puwé| nariéllau adda $<\mathrm{m}>$ pengngeng| Apa' nasitujuangngi| paléléna wenni Botéé| riMa $<\mathrm{m}>\mathrm{pu} \mid$ apa' teppasidapi'pi| ada toMampué nonno'ni Botéé ssawung| Naitani sapposisenna| ana'uréna| Nawaruwanni gelli paimeng| Apa' lisuni paimeng riBoné| Nasipakkedana La Pau $<\mathrm{n}>\mathrm{ru} \mid \mathrm{e}<\mathrm{n}>$ rengngé La Mulia| "Madécéngngi marolata’kua riKajaoé| mappawakkangangngi aléta'| Iamuapasa| méllau adda $<$ m>pangangngi'|"

Apa’ nadapi’ni Itterrung| nagilinna massailé| Botéé| naitani sapposisenna| $\mathrm{e}<\mathrm{n}>$ rengngé ana’uréna| nasenni aléna riola| maélo’ rijallo’ $\mid$ Nassurona palésso ' $\mathrm{i}^{10}$ ulérenna| Aga naseddinni aléna| $\mathrm{La}$ Pau $<\mathrm{n}>\mathrm{ru}$ | dé alepperrenna| majjallo’menni| Sipulireng Botéé| I La Mulia| tau lai-mmanasa ppuliriwi| Riasengngi Botéé| Mati<n>roé rItterrung|

\section{7}

Mati $<\mathrm{n}>$ roéna rItterrung| poana’i $\mid$ Mati<n $>$ roé riGucinna| Bongkangngéna Mangkau'| apa' ripakkaru-mmémengngi| rituona <n>cajiangngéngngi| Tania upomabusung| $\mathrm{La} T \mathrm{Te}<\mathrm{n}>$ rirawé aseng rialéna| Bongkangngé| Bongkangngé pattellarenna| Iana ma’bawiné riTimurung siala Arungngé riTimurung| riasengngé $\mathrm{Te}<\mathrm{n}>$ ripakkiu| $\mathrm{Na}$ dua ana’na| Séuwa ${ }^{11}$ riaseng La Maggalatung| iana maté malolo| Séuwa riaseng Punna riSo $<\mathrm{m}>\mathrm{pa} \mid$ ia ripanguju mattola riTimurung| Maté rijallo'-

10 palésso'i. In the manuscript, two aksara after the pa-have been obliterated and the rest of the word written in above, apparently by another hand.

11 séuwa. The $-u$ - in this word has been neatly added above the line of the text by another hand. Its omission is a clear scribal error. 
[p. 11]

-i I ${ }^{12}$ Da Kalula asenna jallo'éngngi|

Ianaé Bongkangngé| tenrissessia macca| Ripujisia manyame-kkininnawa| Ripuji pa'baruga| Ripujito malempu| Ripujito malabo| Ripujito pasawung| Ripujito maélo' mappasiuno| Riasettoi naéloriwi| séajing mariawana| Riasettoi mmala ada ritomatowanna| Riasengngikia maserro gelli| kkomasai'i

Iatona mangkau'| nataro Tomakkajennangeng| Nakkajennangeng jowana| anakarunna| Joa’ wanuwaé| Anyakkélaié| Sininna poasengngé panré| Sininnato pakkammo makku $<$ n $>$ raié| $\mathrm{pa}<\mathrm{m}>$ puléé| Parala ajué| Pakkeddéé| Pangolo anréngngé| Pallogéé|Iatonana mula engka ballili'|

Ianaé Bongkangngé| Mangkau'| nangka Karaéngngé riGowa| muttama’ riBoné| ssawung nariéwa mattaro| riKaraéngngé| siratu katinna| $\mathrm{Na}$ toPanyula'é séwanuwa| natangkerrangngi| Cella' manu’na Karaéngngé| Bakka'-mattemu manu'na Aru<m>Poné| Nariuno manu'na Karaéngngé| siratu kati rilalettoro'|

Naiatona Mangkau'| namallébu toriAjangale’é| makkatenni riBoné| Nabétai Awo| Téko| nabéta manettoni riAttassalo| Baliéng paimeng|

Iatonaé makkarung nangka Tellumpoccoé| la<n>rengngi babanna riGoa| makkatenni riBoné| naripatudappalili'na Karaéngngé| mmutamai nakkuana riattanna Méru| siduppa Mangkasáé| toBoné| Nattebbanna pitu-ngngesso| Nainappana tauwé| makkeda-<a >da nasiajenna toBoné| toGoaé| Nannessana llao toBoné| wiring riattanna saloé| Tangka nalalo manai'|

Iatona Bongkangngé| Mangkau’ nangka Addatuwang riSompa| riSawitto| naripassu' nalao mai riBoné|

Iatona Mangkau'| nakkappuéng toSoppéngngé rilale $<\mathrm{m}>$ panuwa| Nasaurenna ArusSoppé<n>riaja| ritella'é Mabbéluwa’é| Nalaona mai riBoné| Naiana Mabbéluwaé kkonisa riBoné ma’bawiné| siala ana’daranna Arungngé riBoné| riasengngé $\mathrm{Te}<\mathrm{n}>$ ripauwang $\mid$ Najaji $<\mathrm{a}>\mathrm{nni}$ riasengngé I Dakke’| ritella'é Lébaé| Iatona riaseng Datué riMario| Nallakkaina

12 Matthes appears to have added a pallawa and crossed through the first aksara of this name, thus bringing the text into line with other versions. In his notes, he suggests the form Dakalulla is a contraction of Daéng Kalulla. 
ana'daranna Aru $<\mathrm{m}>$ Poné| tania kupomabusung| riasengngéWéLé $<\mathrm{m}>\mathrm{pe}$ '| siala Maddanrengngé| riasengngé La Saliwu| sappowékkaruamui| Iana ncajiangngi| taniya kupomabusung| riasengngé La Tenriruwa| Matinroé riBantaéng| aseng rimaténa|

Iatona Matinroé riGucié| mangkau' nangka mai mmuttama riBoné| ana’uréna Karaéngngé riGowa| Daéng Pabéta asenna| Ana’uréna Karaéngngé| engkaé mai riBoné| puramani duppai tomajjallo'| Naritellana ritoBoné| Daéng Patobo'| Maddi-Bonéni| Daéng Patobo'| Nangkana Karaéngngé| riasengngé Daéng Bonto| ttériwi Boné| Na kuwana riCellu ssoré| Nammusu'na toBoné| Mangkasa’é| Namalo’na Daéng Bontoipo| $\mathrm{Na}$ lima-ngngesso tauwé matebbang| Nalisu Karaéngngé riwanuwanna|

Naduwa-ttaung purana mammusu' riCellu| nangkasiro mai| Karaéngngé riGowa| ttériwi Boné| Nakuwa riWalenna ttaro bénténg| Nattebbanna toBo-

\section{[p. 12]}

-né| toGowaé| Malo' bessiwi Daéng Patobo'| Nalebbi pitungngesso mattebbang| Nateppani lasa ${ }^{13}$ Karaéngngé| Nariparéwe' riwanuanna| namaténa tengkenne' duwa $<$ m $>$ puleng|

Nangkasiro mai Karaéngngé Daéng Parukka| ttériwi Boné| Nabbalina toriAjangale’é| ia maneng| Naia toTimurungngé| napabétai ana’na| pattaronal nalao mai alau riBonél mádeddessangngi alénal $\mathrm{Na}$ makkunraimani garé riaseng| mmonro riTimurung| silaongngana’na| Naia Lima $<$ m>panuaé| Rilaué| kkoi riCinennung| nawawa pattarona| nauttama mádedderrangngi aléna| woroané riBoné|

Appa’balittoni toriAwamPoné| Nakona riPappolo| Karaéngngé ttaro bénténg| Tépui bénténna| nasosongini Boné| Natelloni Bukaka sépué| enrengngé riTakké Ujung| Apa' lésanni essoé| maélo’ni ma’dimpa Mangkasa'é| Riarupani ritoBoné| Ribuanni Mangkasáé| Nadapi’ni llari Garaéngngé $e^{14} \mid$ Kkui riCempaé| naripeppe’na Mangkasaél Riwettani Karaéngngé| La Turu asenna <m>pettaéngngi|Karaéngngémani riasengngé Daéng Padulung| nattingara toGowaé| Massuroni Karaéngngé

13 lasa. The second aksara seems to have been originally - si, but this has been crossed out and $-s a$ added above in another hand.

14 Garaéngngé. A mistake for Karaéngngé. Another hand has written a faint $\mathrm{Ka}$ - above the first aksara. 
riTallo'| llao riBoné| Naia napoada surona| Karaéngngé riTallo'| "Duwa puammeng| séuwa muwetta ritappéré| séuwa muwetta ritengngana padangngé| Naé maélo’nakkeng ridécéngngé| Téanakkeng rija’é|"

Makkedani Kajaolaliddong| 'Laona makkoniritu adammu| bajapa matu' kuassu’ riKaraéngngé|"

Pappa' bajaé| massu'ni Kajaolaliddong| Kkuni macceppa' Karaéngngé| Napurana sikado-ngngadanna| Purai macceppa'| Karaéngngé riTallo'| toBoné| riasengngé Daéng Padulung| Daéng Patobo’si ripakkarung nriGowa|

Iatona Bongkangngéwé| makkarung riBoné| nasisala Datué riLuwu| riasengngé Sangkaria| Apa' téai paimeng Luwué $\mid<$ m $>$ panuai $C e ́<n>$ rana| Natérisi paimeng Cé<n>rana| Luwué| Agana wékka duwana tanaé riCé<n>rana| riala-bessi ritoBoné| Ianaé| namula ata toUnyi'é riBoné|

Iatonaé Bongkangngé| mangkau' riBoné| nasellao Arung Matowaé riWajo'| riasengngé toUddamang| Nasellaoto Arungngé riSoppéng| ritellaé Pollipué| Appasibuni riCé $<\mathrm{n}>$ rana| kkuni sita masséajing| Iana nassiturusi| passéajingngéngngi <tanana $>\mid$ Nakkeda madécéngngi' sita riTimurung| Nakupa tépu pautta'| ritépunna ulengngé|"

Apa nadapini esso natanraésiduppani riTimurung|Engka manenni toBoné silili'| engka manettoni toWajo'é silili'| engka manettoni toSoppéngngé silili'| Kkuni riBoné ttaro baruga| nakkuna ttaro sawung| Apa' $0<m>$ po' loloni ulengngé| sipulunni toBoné| toWajoé| toSoppéngngé| Situdangenni Aru $<\mathrm{m}>$ Poné| Arung Matowaé riWajo'| Datué riSoppéng| napassiajingngi tanana| iatellu| Kuwaétosia| padaorané sina -ssiama<ng>é| Bonéna macoa| ana' tengngai Wajo| paccucungngi Soppéng| Naina-

[p. 13]

-ppa sitelli'| Naia nassitelliri| “ Tebbaiccukengngé| Tessiacinnangngé ulawettasa'| Pattola malampé| Waramparang malappa'|” Nainappana mallamumpatu| Nasenni tanana Tellumpoccoé|

Ianaé arung maserro riélori| ritoBoné| Maserroto riuddani| Naduwa-ttaung purana mallamumpatu| Tellumpoccoé| nateppani lasa Bongkangngé| Napasipulunni toBoné| "Ia mennang kupoadadakko| anrikku’naritu ttolawa'|" 
Natampaini anrinna| tania kupomabusung| riasengngé La Icca'| Nakkedana| 'Ia kupoadakko-nri'| madodonna'é| Atutuiwi gau'mu| apa' iko ritu kuélorang mangkau’| rékko matéa'| Iatopa kupoadakko| rékko matéa’| naleppe'na winru'-tomatéu'| uwélorangngi mupowawiné ipa’mu| Iomisio maupe'| nangka ana'mu riArut Timurung| Kuélorangngi namupoawiseng| Masulitu makkunrai kkuaé tujunna| enrengngé nawa-nawanna| Nabara' mumuruppengngi tanaé riBoné|"

Purai mappaseng| masialangngi| Riasenni Matinroé riGucié|

\section{8}

Matinroéna riGucié| popadaorowanéi| Matinroé riAddénénna| Ia pawélainana Matinroé riGucié| tania kupomabusung| La Icca’na makkarung riBoné| apa' ia ripasengngang rikakana| Naia purana nawinru' tomaténa| Matinroé riGucinna| rilanti'ni|

Purai rilanti'| napowinéni Arungngé riTimurung| Tenripakkiu| Najajina riasengngé| tania upomabusung| La Tenripale' To Akke $<\mathrm{m}>$ péang pattellarenna| Najajitona| tania kupomabusung| Wé Tenrijello'| Iana ritella Makkalaru'é| Engkatopa séuwa| malolomupa namaté|

Iana Mangkau' riBoné| tania upomabusung| riasengngé La Icca’| nangkaro mai Karaéngngé riGowa| ttériwi Boné| Naé tellattu’toni nalisu| Karaéngngé|

Ianaé arung| tania kupomabusung| tenrisseng rinawa-nawa| Iamua naripauttama risure'é| annessanamua gau'é|

Ianaé Mangkau'| nammulana toBoné| tessiseng siéwa ada| Nagillinna ${ }^{15}$ ArupPallenna| riasengngé La Panaongi| To Pawawoi pattellarenna| Naripalina kuwa riSidénréng| Apa' mangingngini ttudang riSidénréng| maélo’muani llisu riBoné| méllau addampeng| Risurosi maddé' llao manai' riBuki'é| Riolani nariuno| ArupPallenna Riunotoi|Arungngé riPaccing Riunotoi| Arungngé riAwamPoné riasengngé To Saliwu| Riawang Riunotoni| Maddanrengngé riPalakka| riasengngé To Saliwu Riwawo| Maégato arung toBoné| riuno| Nasalossoni tau tessalossorennaépa| Napaténi tau teppatérennaépa| Onconni tessisenna siéwa ada toBoné| naé' dépa gau’|

15 Nagillinna. A mistake for Nagellinna. 
Iamua garé nawinru wette'reng| Engka séuwa esso| nangka toBoné| nalaong naré'-aréi awisenna| Naridapi'na namaélo'na tauwé

[p. 14]

$-<$ m>punoi| Nalarina tauwé| Iamani wawinéna tauwé nauno| Nainappana massellang| Napanréiwi api sipué Boné| gangkanna Matajang| lattu' urai'| riMacégé| Natasséa-séana| toBoné| Iamani| arung <e>ngkapa| $<$ m>pokke'ngaléna| nalao maniang riMajang| Makkedani Puatta’| riMajang| "Maragotu mai|"

Makkedai toBoné| "Tekkisseng palappa-lappai Puang| Tellommunosa ${ }^{16}$ muitai monorang| wanuwaé riBoné|"

Apa' tellonni Puatta riMajang| nata $<\mathrm{m}>$ puini arona nakkeda| "Malebborang énnajai lumuna Puakku'|" Naé dépa adammeng| napoada toBoné| "Assurosao mmalangnga' riMa $<\mathrm{m}>\mathrm{pu} \mid$ anauréku| riasengngé Da Malaka| Apa' iamenniritu| Arung Matoa|"

Makkedai tauwé| "Engkamui monorang riPalakka Puang|"

Rilaona rialai| Temmai<tta>to nangkaro mai| Makkedai Da Malaka| "Aga Puang tassuro mmalangnga'|"

Makkedai Puatta' riMajang| "Iatu llaomu romai| Temmuitaga| rumpu apié riBoné|"

Makkedaa ${ }^{17}$ Da Malaka| "Nasia Puwang| uwitamua|"

"Ianatu| uwassuro mmalako| pékkoni| nawa-nawammu|"

Mekkomoi| Da Malaka| Nawékka-tellumana| riéwa ada| riPuatta' riMajang| nainappana makkeda| "Matau'ka' Puang| Madécé-mmua| rékko passesse’mua| limpoa'|"

Makkedai| Puatta' riMajang| "Idi' sikuwaéwé| Tagerro séuwamuni| tauru|" Makkedani Da Malaka| “Makkoniritu adatta' Puwang| Naé” dé’ salai| nadapi' nawa-nawaku'| Iamani| tapassu'é nalengngi'ang lebbi'|na tanaé| Namau puatta' riolo| nalebbiranga-mmémessa tanaé| nawatakkaléna| maui tennapoade'| mappassué| Ia’nasia| mulai gau'| kupassui anauréu'|"

16 Tellommunosa. The second aksara has been added above the line, apparently by the original scribe.

17 Makkedaa. A mistake for Makkedai. 
Jaji Da Malakana| patetto-ngngada| Nasuroiwi ana’uréna| makkedaé| "Assuko| tanikoritu sengngangke’ tanaé|"

Nalattu'na torisuroé| Kkumoi ritanaé| napoléi| napalattu’ni risuroangngéngngi| Tennapoada manettopa| nariunona|Suroé| Nainappana| tello manengngi bolaé| riBoné| riLalebbata|

Aga paissenni| Puatta' riMajang| déna bola riLalebbata| Makkedani Puatta’| riMajang| "Uléka'| kalaki'| muwawa riBoné| naia' llao| sipulireng eppou’ Makkullénaritu| uwéwa siuno| Laona| taniana arung|"

Makkedai Da Malaka| 'Laotoa' matu’| apa' pura manenni| mattaro ada| tagerro séuwa taurui"|

Lao manenni tauwé| riBoné| Ripoléiniria| iamani rialé-aléna| Inappai naita tau tebbéé| nalluruini| maéga tau tauno ${ }^{18} \mid$ Makkomaniro garé| gau'na Iasi nalurui| iasi llari| Ia nabokori| iasi mmolaiwi| narang puru'na

[p. 15]

Llaomuni riaddénénna| ssanré| Nalaona Puatta’ riMajang| sila’i| ulunna| eppona| namaténa| Nariasenna Matinroé riAddénénna| Makkedatoi taué| To $<$ m>pawaéngngi| Salassana| Seppulo séuwa| taunna mangkau’| iamatona| namaté|

\section{9}

Matinroé riAddénénna| sapposisengngi Matinroé| riBettung| Ia maténana| Matinroé riAddénénna| sipulunni| toBoné| riPuatta’ riMajang| nasipattangngareng| naningngarén na| tapatetto-ngarung|

Napatettonna élo'| Arungngé riMajang| Makkedai| Arungngé riMajang| "Nalaleng iagasa tala arung| tenna eppoo ${ }^{19}$ La Pattawe'| ana'na ArupPallenna| eppona Makkale $<\mathrm{m}>$ pié|"

Najajina samaturu' Arung toBoné| kkado| ArukKajuna| ripatettong| Arung| riBoné| nariasenna| ArumPoné| Iana riaseng| La Pattawe'| Nabbawinéna| ArumPoné| siala Arungngé riMampu| Nanảna| taniya upomabusung riasengngé Tenritappu|

18 tauno. A mistake for nauno.

19 eppoo. A mistake for eppou'. 
Nabbawinéna| tania upomabusung| La Tenriruwa| siala massapposiseng| riasengngé Dakke'| Najajiangngi| tania upomabusung| riasengngé La Tenrisui| Naé dé ridapi'| riéngkalinga| paunna rilale-ngakkarungenna| Iamua makkedaé| pitu-ttaummui| mangkau' riBonél Nalao riBuluku $<\mathrm{m}>\mathrm{pa} \mid$ nako nateppa lasa| naiamatona| mpawai nasiasenna

\section{0}

Matinroé riBettung| Poana’i| Matinroé riSidénréng| Apa' iana| ana’na Matinroé riBettung| riasengngé $\mathrm{I}$ Tenriu $<\mathrm{m}>\mathrm{pu}$ | iana riaseng| Aru $<\mathrm{m}>$ Poné|

Ianaé mangkau'| napatettong ArupPitu| Naia Matowaé| riTobojong ${ }^{20} \mid$ riasenni Arut Tibojong| Matowaé riTa'| riasenni ArutTa'| Matowaé riUjung| riasenni ArungNGujung| Matowaé riPoncéng| riasenni ArupPoncéng| Matowaé riTanété| riasenni ArutTanété| Matowaé riMacégé| riasenni ArumMacégé|

Makkedai| Aru $<$ m>Poné| "Ia mennang| kupatettommu| ArupPitué| maélo’mua'| muampiri| rilao $<$ n $>$ rumaé| enrengngé ritoanana| arungngé riBoné| apa' makkunraia| Enrengngé maélo’ka’| musapparang lise’na salassaé| tatteré-teré| Naékia| upatettommu ArupPitu| temmulawiriwi| tanaé riBoné| temmuaddi $<\mathrm{m}>\mathrm{pa}$ sunrang| temmamanako riana’mu| rékko tekkuissengngi| sangadinna rékko situdangengngi'| idi'maneng| wijanna Mappajungngé| nasituru’ makkarungngé riBoné| Nappasisa llao| inappatonisa| llalo pa-

[p. 16]

-mmanamu| riana'mu| rieppomu|"

Iatonaé| makkarung riBoné| nangka Karaéngngé| riGowa| ttérang asellengeng| Ajatappareng| napaolai| osong| Nalao Tellu $<\mathrm{m}>$ poccoé| duppaiwi| Mangkasa’é| laona ssu'| Karaéngngé| riwanuwanna|

Nasitaummua| nangkasi Karaéngngé| ttériwi Pandang-Pandang| Nalaosi| Tellu $<\mathrm{m}>$ poccoé| Nasiduppa rilau'na| Bulu' Sitoppo'| nasiabbaléccorang| tauwé| Naribuanna| Tellu $<$ m>poccoé| Napada-llaona| riwanuwanna| narussa'na| atTellu $<\mathrm{m}>$ poccongengngé| Boné| Wajo| Soppéng| 
Nasétaung| marussa' Tellu $<\mathrm{m}>$ poccoé| nangkasi Karaéngngé| ttériwi Soppéng| Tennalaona| toBoné| toWajo’é| bbaliwi toSoppéngngé| Naribétana| Soppéng| nasellenna toSoppéngngé|

Nasétaung| sada’na| toSoppéngngé| nangkasi Karaéngngé| ttériwi Wajo| Nanga $<$ n $>$ rona to Wajo'é| naripauttamasi sada'|

Nasétaung purana| sada'| toWajo'é| nalao Aru $<\mathrm{m}>$ Poné| riSidé $<\mathrm{n}>$ réng| maélo' makkutanangngi| akkuwa-uwanna asellengengngé| Nalattu’mua riSidé< $<$ réng| nasellenna| Nakotona riSidé< $<$ réng| nateppai lasa| $\mathrm{Na}$ iana $<$ m $>$ pawai| Nasera ${ }^{21}$ taunna| Mangkau' riBoné| namaté| Nariasenna| Matinroé riSidénréng|

\section{1}

Mati $<\mathrm{n}>$ roé riSidénréng| sapposisengngi| Mati<n $>$ roé riBantaéng| Ia pawélaina| Mati<n>roé riSidénreng| sipulunni toBoné| Naia nassiturusi| ArupPalakka narutto riPattiro| apa' eppona wali-wali| Mappajungngé| Mappajungngé| ArupPalakkana| ripasekkoreng| pajung| Tania upomabusung| La Tenrirua| aseng rialéna| Iatona| ripésonaiang| alé| ritoBoné| paoppang|paléngengngi| tanaé riBoné|

Apa' tekkenne’topa| tellu $<$ m>puleng makkarung| nangkana Karaéngngé| ttériwi Boné| riMusu' Sellengngé| Ma’bénténni| riCellu| Mangkasa’é| ma’béntéttoni| riPalletté| karaéngngé| Rita $<\mathrm{n}>$ réréanganni asellengeng| toBoné|

Makkedai Aru $<\mathrm{m}>$ Poné| “Laona ia mennang| mupésonaia'| paoppang|paléngengngi tanaé riBoné| mupasekkori pajung| Naé nata $<\mathrm{n}>$ réréangangngi’ décéng| Karaéngngé| Madécéngngiasa| taceppa’| asellengengngé| $\mathrm{Apa}$ ia| uluadatta’| riolo| riKaraéngngé| ia llolongang| décéng| tajang| ia mappaita| Nakkedana Karaéngngéwé|'Uwasengngi décéng| tajang| makkatennikku'| riagamana| Na’bié| Nakkedatopa Karaéngngé| Rékko mutarimai| adakku’

21 Nasera. Matthes has corrected this to Naséra. 
[p. 17]

dua maraja| Gowamua| naBoné| tapadana makkasiwiang| riDéwata Séuwaé|" Makkedatopi| Aru $<\mathrm{m}>$ Poné| "Rékkoromi| tettaririmai ${ }^{22}$ $\mathrm{a}<\mathrm{da}>\mathrm{ada}^{23}$ madécénna| Karaéngngé| tatongengiwi| natelloppi’ matu| nanyo $<\mathrm{m}>$ pa| atani'| asenna| Narékko tatarimai| ada madécénna| Karaéngngé| nawélaiyyangngi' ada| matti parimeng| muasengnga’| téa méwai| siwuno| Inappatonisia| kuéwa| rékko nawélaiangnga'| ada|"

Samatéani| toBoné| kkadoiwi| asellengengngé|

Mekko'muni| Aru $<\mathrm{m}>$ Poné| apa' nase'dinni| Aru $<\mathrm{m}>$ Poné| lainna| kédona toBoné| Nalaimuni| aléna Aru $<\mathrm{m}>$ Poné| nalao riPattiro| Tau rialénamani| mmolaiwi| Apa' llattu'i| riPattiro| naéwasi ada| toPattiroé| téamessi mmolaiwi| asellengengngé| Mekko’muni Puatta’| naté’ risalassa’é| ma'dedde'| Rangeng rialéna|tomanisa| nasilaongang| Anảna| pattarona| napaté maneng| risalassa'é|

Naia lésso'na| llao riPattiro| Aru $<m>$ Poné| sipulunni toBoné| Ia nassiturusi| passu'éngngi| Aru $<\mathrm{m}>$ Poné| Nassurona toBoné| llao riPattiro| na To Alaung| asenna| risuroé| Nalattu'na| To Alaung| riPattiro| téni manai'| risalassa'é| Makkedai| "To Alaung ngAmang| Ia nasuroangnga'| toBoné Amang| Tanidi’naritu| téaio| idi’naritu| téaikkeng| Natujuni bali| <a>tammu| riBoné| muwélaiwi|"

Makkedai Puatta'|“OTo Alaung| massakkakoitu| téawi toBoné| uwélorinna muaritu| toBoné| Kuripaitai décéng| e $<$ n $>$ rengngé tajang| kumaélo'tongenna| ré $<\mathrm{n}>\mathrm{ré} k \mathrm{ko}$ ritajangngé| mutéa mennang| Naé akkatenninno| mi| rinawa-nawa pettammu| Ulaotonasa| ritajanna paranyalaé| Déwata Séuwaé| riNa’bié”|

Purai makkeda| To Alaungeng| $<$ n>réwe'ni riBoné| Iasi nassiturusi| toBoné| ArutTimurungngéna| napakkarung riBoné| Apa' ana’nai| Mati<n>roé riAddénénna| tania upomabusung| riasengngé| La Tenripale’| aseng rialéna| To Akke $<\mathrm{m}>$ péang| aseng $<\mathrm{n}>$ riana’na| Pawélaimenni| nariasenna| Mati $<$ n $>$ roé riTello'| Iana makkarung| naparéwai| toBoné| rimusu sellengngé|

Naia| lésso’na nalao| To Alaungang| llao riBoné| massuro ma-

22 Tettaririmai. The copyist seems to have begun the aksara for the second -ri- as a -ma-, but then corrected it to add emphasis.

$23 a<d a>a d a$. The repetition of the word seems to be intended. 
[p. 18]

-tonisa| Puatta'| llao riKaraéngngé| koriPalletté| Lattu’i riPalletté surona| massurotonisa| Karaéngngé| llao riPattiro| Karaéng Pettung| risuro| Aga lettu'nana| Aga lettu'nana ${ }^{24}$ | Karaéng Pettung| riPattiro| ripasiléwoni| Puatta'| ritoPattiroé| e $<$ n $>$ rengngé ritoBulu'é| Naruppaini| najjallo'| Nabuanni| Sébulu'é| e $<$ n>rengngé toPattiroé| Napakkappoi| riBulu’é| riMaroanging|

Puraikuwa| malliwenni| riPalletté| Puatta’| sita Karaéngngé| Karaéngngémenni| Pettung| mon $<\mathrm{n}>$ roangngi Pattiro| Aga lettu'ni| Puatta’| riKaraéngngé| makkedani Karaéngngé| "Madécénnibéla| laona| llaoko mai| Naiasa| ukkutana riko| kéga| gangkanna| anurialému| Mauékko temmakkarung| riBoné| mupoanumua| Apa' uwaseng| mupoanu Boné| naé léléni|"

Makkedai Puatta’| "Anu rialéu’na| kuwaé Palakka| $\mathrm{e}<\mathrm{n}>$ rengngé Pattiro| e $<$ n $>$ rengngé Awa $<$ m $>$ Poné| Naia| Mario-Riwawo| anu rialénatosa| awisekku'|"

Makkedai Karaéngngé| “Sada’no| naiatonasotu| sékkuwaé| mutama’ sada’| mupoanué| Boné teppoatao| Goa teppoatao|”

Makkedai Puatta'| "Sada’é mémenna'| Karaéng| kulao mai|"

Nainappasi makkeda| Karaéngngé| "Uwissemmua| mupoanu Palletté| Naé tettongennai| batéu'| uwasenni anukku'| naé <a>nukku’nisia| Palletté| kuwérékko"|

Nainappasi| riwéréng Puatta’| riKaraéngngé| appala baludu’| rikoi' ulawettasa'| sikati berre'na|

Makkedai Puatta'| "Rékko ia Karaéng| muwéréngnga'| tekkusilaonganna| toBoné| mméwao| téawa’ mmalai|”

Makkedai Karaéngngé| "Muisse $<\mathrm{n}>\mathrm{ritu}$ baiseng| ade’nai torioloé| rékko sitai passéajingenna| engkatosia| nawi $<\mathrm{n}>\mathrm{ru}$ ' assitang| sullé alosi séire'| éwulu sila $<$ m>pa|"

Makkedai Puatta'| "Uwanuritu| Karaéng| lanako adammu”| 
Puraikuwa| inappani| makkuluada| Puatta'| Karaéngngé| Puatta' Mati<n>roé| riBantaéng Karaéngngé riGoa| Mula Sellengngé| Karaéngngé riTello'| Mula Sellengngé|

Ianaé| akkuluadangenna| Makkedai Karaéngngé| "Iana tappasabbiang [p. 19]

riDéwata Séuwaé| taniapa wijammeng| makkarung riGowa| riTallo'| temmupoanui anummu| murigau' bawang| ripadammu tau| Naé rékkua engka ja'| ttujuo| ti<m>pa'i tange'mu| kutama rija'mu|"

Makkedatonisia| Mati<n>roé riBantaéng| "O Karaéng| temmarunu' wesséu'| tessekka bilakku'| tenriti<m $>$ pa' balawo rita $<$ m $>$ pukku'| naé dékkuwa| engka tujui| tanaé riGowa| Mau sébatammua awo| uwappangi kulao| matturi ssu'| riperri'mu Karaéng| Lettu' ritorimunrimmu| ritorimunrikku|tosa| rékkowa tenriwélaia-mmuakkeng ada| rikkeng tobaiccu'é|"

Kkunié akkuluadanna| Matinroé riBantaéng| na Karaéngngé| Purai macceppa' Puatta’| na Karaéngngé| lisuni paimeng riPattiro| $\mathrm{Na}$ lima $<\mathrm{m}>$ penni| purana macceppa'| Karaéngngé| na Puatta' Matinroé riBantaéng| natello Boné| narimusu' sellengngé| Manyo $<\mathrm{m}>$ pani toBoné| naripasada’na|Nalisuna Karaéngngé| riwanuanna|

Nalésso'mua llao Karaéngngé| narisurona meddé| Matinroé riBantaéng| ritoBoné| Nalaona ssu' riMangkasa| Naana' riDato’ Bandang| Nai aseng Jawana| Matinroé riBantaéng| riDato' Bandang| Adama aseng Jawana| Matinroé riBa<nta>éng|

Maittai mmonro riDato' Bandang| Ripangiléni onrong| riKaraéngngé| Naia naélori| naonroi| riBantaéng| Agana kkuna riwawa| $\mathrm{Na}$ kuna mmonro| Matinroé riBantaéng| nakkuna narapi' umuru'na| nariasenna Matinroé riBantaéng| 
Matinroé riBantaéng| ripassu'| sapposisengngi Matinroé riTello’| Iana ripassu'na Matinroé riBantaéng| ArutTimurunna| nassiturusi toBoné| napakkarung| Apa' ana'na Matinroé riAddénénna| riArungngé riTimurung| Ianaé Aru $<\mathrm{m}>$ Poné| tania upomabusung| riaseng $\mathrm{La}$ $<$ Ten $>$ ripale'| To Akke $<$ m $>$ péang pattellarenna|

Ianaé mangkau’| naparéwe’i toBoné| riMusu' Sellengngé| Naritellona Boné| nanganrona toBoné| tenriala| sebbukatinna| tenriéllau rebbabaténa| tenrirappatoi $\mid$ Manyo $<$ m>pana toBoné| ripasada’muni napura| Naiakia tudappalili'manenni|

Naia puranana ripasada’ toBoné| ripasada’ sépalili'| laotonisa Karaéngngé| riwanuanna| ArutTimurunna| mangkau’ riBoné| Pawélaimani| nariasenna Matinroé riTallo’

Naé| duwai sijajing| Matinroé riTallo'| Anrinna riaseng| tania upomabusung| Wé Tenrijello'| Iana ritella Makkalaru’é| Iana Makkalaru’é| sia-

[p. 20]

-la Arungngé riSumali| riasengngé La Pancai| Najajina riasengngé| La Maddaremmeng| Salih aseng Jawana| Iana ripakkarung riTimurung| Nariala Pattiro| iatona Mappakkalaru'é25 Arung riPattiro| Séuwatopa anrinna| tania kupomabusung| La Maddaremmeng| riaseng $\mathrm{Te}<\mathrm{n}>$ ria $<\mathrm{m}>$ pareng| Iana makkarung riCellu| Séuwatopa anrinna| riaseng La Tenriaji| To Senrima aseng riana’na| Iana ripakkarung riAwamPoné| Iatona riaseng| Pawélaié riSiang|

Nallakkaina| tania upomabusung| riasengngé Wé Tenrisui| siala La Pottobune'| arungngé riTana-Tengnga| Najajianni riasengngé| Da Unru| tania kupomabusung| riasengngé La Tenritatta| To Unru' aseng riana’na| tammana'| Enrengngé| Da Tenrigerra| puttatoi| e $<$ n $>$ rengngé $\mathrm{Da} \mathrm{O}<\mathrm{m}>$ po' tammana'| $\mathrm{E}<\mathrm{n}>$ rengngé $\mathrm{Da}$ Émba| Enrengngé riasengngé| tania kupomabusung| riasengngée ${ }^{26} \mid$ Wé Pappolobonga ${ }^{27} \mid$ Da $U<m>$ pi aseng riana’na| Iana riaseng| Madda $<\mathrm{n}>$ rengngé|

25 Mappakkalaru'é. This should be Makkalaru'é as twice previously in this paragraph.

26 riasengngé. This repetition is unnecessary.

27 Pappolobonga. She is usually known as Mappolobombang. 
Nasétaung purana ssada'| toBoné| nalao ssu'| riMangkasa| Nasita| Dato' riBandang| riasengngé| Abeddulla| aseng Jawana| Aru $<\mathrm{m}>$ Poné| Iana Arung manyame-kkininnawa| Riasettoi pabbaruga| riasettoi maserro pallao $<$ n $>$ ruma

Iana siala| ana’na Matinroé riSide $<$ n $>$ réng| ritellaé| Kaunangngé| Ana’na ritellaé Dabe'| Dabe'na situmaé| anảna Karaéngngé riGowa| Mula Sellengngé| Daéng Mattola asenna| ana’na Karaéngngé| potumaiéngngi| Dabe'| Naé tennawette'pa dara| namatél Jaji déna ana’padana| Aru $<\mathrm{m}>$ Poné|

Ianaé| arung mallao-lisu riKaraéngngé| maittawégangngi| rékko telluttaungngi| nalaosi ssu'|

Apa' nasitujuangngi| llaona ssu'| riMangkasa| lattu’i ssu'| riMangkasa'| kuani nateppa lasa| iana lasa $<$ m>pawai| Agana kuana riTallo'| rilemme'| nariasenna Matinroé riTallo'| Dua-ppuloi taunna| mangkau'| napawélai|

\section{3}

Matinroé riTallo'| napoanauré riwakkangngi| Matinroé riBukaka| Ia pawélainna| Matinroé riTallo'| ana'uré riwakkannana ttolai| mangkau' riBoné| Apa' ia nappasengngang| tania upomabusung| La Maddaremmeng| aseng rialéna| $\underline{\text { Salih }}$ aseng jawana| Pawélaimani| nariasenna| Mati $<\mathrm{n}>$ roé riBukaka|

Iana Mangkau’| nawinru' Paju<m>puté|

Iana ma’bawiné riWajo| siala| Hatija| Da Se $<$ n $>$ rima| aseng riana’na| ana’na Arung Matoaé| riWajo'| ritellaé| To Ala’é| Céuwamua| ana’na| Aru $<\mathrm{m}>$ Poné| iamua ria-

[p. 21]

-sengngé Pakkoko’é| To Akkonéng pattellarenna|

Iatonaé makkarung| napalowangngi bataé riBoné| napangésa'-alaui| napangésa'-maniangngi| 
Iatona riaseng| masse' magama| Iatona massola| iagelli temmassolaé| Nasuroni massolo ncajiangngéngngi| naia napoada| Mappakkalaru'ée ${ }^{28} \mid$ naia napoada| "Temmakkullésa'| temmassuro-suro"

Nagillinni ncajiangngéngngi| natérini Pattiro| narappa-rappai| Llarini ssu'| Mappakkalaru'é| riKaraéngngi| Massuroni Karaéngngé riGowa| pakainge'i

Aru $<\mathrm{m}>$ Poné| Ritérisi riKaraéngngé| naribétasi Boné| Nabéta manai'| riCe $<\mathrm{m}>\mathrm{pu} \mid$ Aru $<\mathrm{m}>$ Poné| Nariolang| naripallaleng| riwawa ssu' riMangkasaé| Nakkuna riSiang| ritaro|

Seppulo-lima-ttaunna| makkarunna| nabéta toBoné| Pawélaimani| nariaseng Matinroé riBukaka|

Naia ribétana| Boné riMatinroé riBukaka| pada-oroanénasi| Matinroé riBukaka| riasengngé To Se $<$ n $>$ rima| mmonro riBoné| Naritérisi paimeng| riKaraéngngé | Naribétasi Boné| narilaling| Iana riaseng| Béta Pasé $<m>$ pe’| apa' kkoi te' riPasé $<\mathrm{m}>$ pe'| maréwang toBoné| Nariwawatona ssu'| To Senrima| nakkuanaria maté| Nariasenna Pawélaié riSiang|

Matinroémani riBukaka| mmonro riSiang| silaong toBoné rilalingngé| Ma’gangkasanié ripau| Mati<n>roé riBukaka| rionronnapasi naripau| apa' déna arung riBoné|

Ga<ng $>$ kannamani| najennammani napatettong| Karaéngngé| mmonro riBoné| To Bala| asenna jennangiéngngi Boné| Mannessani llao atana| tauwé riBoné| riMangkasa’é|

Naseppulo-pitu-taunna| Jennang To Bala| naparéwai toBoné| Naribétasi toBoné| riMangkasáé| Nariwettana To Bala| Iana poasengngi| Béta To Bala| 
Nalliwenna riButung| Matinroé riBontoala'| Naé' ma'gangkasaniro ripau| akkatangenna tanaé riBoné| riMangkasaé|

Ia maténa To Bala| ArungNGamalisi Jennang riBonél Napituttaung ArungNGamali| nawawai To Boné| llao riButung| Na kuna riButung| toBoné| nangkatonaro mai| Matinroé riBontoala'| silaong Balandaé| Narialana toBoné| riMatinroé riBontoala’| ripasiala Karaéng Bontomarennu| enrengngé Mangkasaé| iamaneng|gangkanna engkaé riButung| Tammat 
This text is taken from The Bugis Chronicle of Bone, translated and edited by Campbell Macknight, Mukhlis Paeni and Muhlis Hadrawi, published 2020 by ANU Press, The Australian National University, Canberra, Australia.

doi.org/10.22459/BCB.2020.06 